\title{
Semi-Active Adaptive Fuzzy Sliding Mode Control of Buildings under Earthquake Excitations
}

\author{
Amir H. Rabiee, Amir H. D. Markazi \\ School of Mechanical Engineering, Iran University of Science and Technology \\ Narmak, Tehran 16844, Iran \\ ah_rabiei@mecheng.iust.ac.ir; markazi@iust.ac.ir
}

\begin{abstract}
New Adaptive Fuzzy Sliding Mode Control (AFSMC) method is proposed for semi-active control of buildings under earthquake excitations. The proposed method has the important advantage of being model-free, and therefore, can cope with uncertainties in the structural model, high nonlinearities in the behaviour of MagnetoRheological (MR) dampers and the random nature of the earthquake excitations. The proposed approach encompasses a fuzzy system and a robust controller. The fuzzy system mimics an ideal sliding-mode controller, and the robust controller compensates for the difference between the fuzzy controller and the ideal one. The parameters of the fuzzy system, as well as the uncertainty bound of the robust controller, are tuned adaptively. The adaptive laws are derived in the Lyapunov sense to guarantee the asymptotic stability of the controlled system. The AFSMC controller determines the needed control force, and an internal force-following loop approximately generates the required interaction force by intermittent activation of the semi-active dampers (Clipped algorithm). A three-story benchmark building with only one MR damper in the first floor is considered. By comparing the structural responses in the uncontrolled case, AFSMC/Clipped system and an H2-LQG/Clipped control strategy, advantages of AFSMC method are demonstrated.
\end{abstract}

Keywords: AFSMC, Clipped algorithm, semi-active control, MagnetoRheological dampers

\section{Introduction}

In order to save lives and reduce the costs associated with the hazards of earthquakes and strong winds, many efforts have been reported in the literature and also physical implementations to alleviate structural vibrations under such natural disasters. Depending on the type of employed control devices, control methodologies are classified into active, passive, hybrid and semi-active systems. Among these methods, the semi-active methodology is of more interest, because it can provide comparable performance to the active methods, yet exerting energy to the building almost as small as passive methods. This is particularly important, because the risk of electrical power loss in the event of devastating earthquakes is extremely high.

MagnetoRheological (MR) dampers are probably the most commercial and popular semi-active control devices used for vibration reduction of buildings and advanced vehicles. In general, previously proposed control strategies applicable to MR dampers may be divided into two categories: (1) Model based approaches and (2) Heuristic or intelligent methods. In the model-based control strategies, exact mathematical models of the building and MR dampers are needed [1-3]. However, since the modeling process of buildings and, in particular, MR damper involve simplifications and un-modeled dynamics, i.e., uncertainties, the performance of the resulting control models may be negatively effected in practical implementations. A particular complication, compared to the active methods is that the physical behaviour of MR dampers is highly nonlinear and depends on the dynamic interaction with the associated structure. Intelligent control approaches are generally divided into neural network, neuro-fuzzy and fuzzy logic based control methods. In particular, fuzzy controllers have been used extensively for the control of structural vibrations. Originally, tuning of fuzzy membership functions and fuzzy rules were performed through cumbersome trials-and-errors. The inadequacy of conventional methods motivated new optimization algorithms, such as neural networks and the genetic algorithm $[4,5]$. Due to the unpredictable nature of earthquake excitations, however, non-adaptive methods may not lead to an acceptable performance. Wang [6] introduced the Adaptive Fuzzy Logic Control (AFLC) strategy, including an online adjustment method for fuzzy parameters, in order to enhance the performance of conventional FLC. In this strategy, the adaptive nature of the controllers eliminates the need for advanced attainment of the system's dynamic characteristics. Furthermore, as the controller parameters can be 
tuned on-line, the fuzzy rules can be roughly specified, or even entirely unspecified in advance. On the other hand, the Sliding Mode Control (SMC), which is known to provide excellent stability, robustness with respect to uncertainties and external disturbances [7], could lead to degraded steady-state performance. In particular, extremely rapid switching with large control gains on the sliding surfaces could cause unacceptable high-frequency chattering in the control signals. The chattering problem can be resolved by applying fuzzy control signals in place of switching functions. As a further control performance improvement, AFLC approach may be presented into SMC to arrive at the hybrid adaptive fuzzy sliding mode control (AFSMC) strategy [8,9]. The discontinuous switching function and uncertainty terms of the equivalent control are replaced by fuzzy system to reach condition of the sliding mode control and mitigate the chattering phenomenon as well as the detrimental effects caused by uncertainties. The on-line tuning of fuzzy parameters, together with online estimation of model uncertainties provides the simultaneous advantages of both AFLC and SMC methods.

In this article, the AFSMC is employed for semi-active control of buildings. The proposed approach encompasses a fuzzy system and a robust controller. The fuzzy system mimics an ideal sliding-mode controller, and the robust controller compensates for the difference between the fuzzy controller and the ideal one. The parameters of the fuzzy system, as well as the uncertainty bound of the robust controller, are tuned adaptively. The adaptive laws are derived in the Lyapunov sense to guarantee the asymptotic stability of the controlled system. The AFSMC controller determines the needed control force, and an internal force-following loop approximately generates the required interaction force by intermittent activation of the semi-active dampers (Clipped algorithm). A three-story benchmark building with only one MR damper in the first floor is considered. By comparing the structural responses in the uncontrolled case, AFSMC/Clipped system and an H2LQG/Clipped control strategy, advantages of AFSMC method are demonstrated.

\section{Adaptive fuzzy sliding mode control}

Sliding Mode Control (SMC), is known to provide good stability and robustness for control of nonlinear systems. Since conventional SMC could lead to unacceptable chattering control signals, it is mimicked by a fuzzy controller. Online tuning of fuzzy parameters, together with online estimation of model uncertainties, provides the simultaneous advantages of both SMC and AFLC methods.

\subsection{Sliding mode control}

The designing procedure of a conventional sliding-mode controller includes two steps: the first is to find a feedback controller which causes the system state trajectory to reach the sliding surface $s$ in a finite time and remain there; and the second is to guarantee that the obtained trajectory on s is stable using a switching action.

Consider the $\mathrm{n}^{\text {th }}$ order system that can describe the behavior of building as:

$$
x^{(n)}=f(\mathbf{x})+g(\mathbf{x}) u
$$

where $\mathbf{x}=\left[x, \dot{x}, \ldots, x^{n-1}\right]=\left[x_{1}, x_{2}, \ldots, x_{n}\right] \in R^{n}$ is the vector of system states which are assumed to be measurable, $u \in R$ is the control input, $f(\mathbf{x})$ and $g(\mathbf{x})$ are smooth functions, which are unknown.

The tracking error is defined as:

$$
\tilde{x}(t)=x_{d}(t)-x(t)
$$

where $x_{d}(t)$ is the desired trajectory of system states. The goal is to determine a controller for the system Error!

Reference source not found. which can make the tracking error converges to zero, i.e. $\lim _{t \rightarrow \infty} \tilde{x}(t)=0$.

In case of having uncertainties, the system Error! Reference source not found. becomes:

$$
x^{(n)}=f(x)+g(x) u+d
$$


where $d$ is the lumped uncertainty which is assumed to be bounded as $|d| \leq \delta$. By using the time derivative operator $D=d / d t$, the sliding surface becomes:

$$
s=(D+\lambda)^{n-1} \tilde{x}
$$

with $\lambda>0$ a user defined constant. The sliding mode control law is defined as the following:

$$
u=u_{e q}+u_{r b}
$$

where the equivalent controller $u_{e q}$ is a feedback linearization controller, obtained from $\dot{s}=0$. And the robust controller $u_{r b}$ is designed for compensating the uncertainties, as:

$$
u_{r b}=g^{-1}[\delta \operatorname{sgn}(s)]
$$

By considering the candidate Lyapunov function $V=\frac{1}{2} s^{2}$, the SMC system, guarantees the stability of the unknown systemError! Reference source not found. [8, 9].

\subsection{Fuzzy approximation of sliding-mode control}

Now, assume that the parameters of system are known completely. In such a case, the ideal controller can be obtained as $u^{*}=u_{e q}$. From practical point of view, the parameters of system may not be specified exactly; so the ideal controller cannot be determined according to Eq. Thus, by using the universal approximation capability of fuzzy systems $u^{*}$ can be approximated using a fuzzy system.

Consider a TS fuzzy system with input $s$ and output $u_{f u z}$ with $n_{r}$ fuzzy IF-THEN rules, as:

$$
\text { Rule } \mathrm{r} \text { : If } s \text { is } \tilde{A}^{r} \text { then } u_{f u z}=\tilde{b}^{r} \quad r=1, \ldots, n_{r}
$$

Then the output of the fuzzy system can be written as:

$$
u_{f u z}(s, B)=B^{T} W
$$

where $B=\left[\tilde{b}^{1} \ldots \tilde{b}^{n_{r}}\right]$ and $W=\left[w^{1} \ldots w^{n_{r}}\right]$. Therefore the ideal controller $u^{*}$, can be approximated by an ideal fuzzy system $u_{f u z}^{*}\left(s, B^{*}\right)$ and is equal to:

$$
u^{*}=u_{f u z}^{*}\left(s, B^{*}\right)+\psi=\hat{B}^{* T} W+\psi
$$

where $\psi$ is the approximation error or the uncertainty, which is assumed to be bounded as:

$$
|\psi|<\Psi
$$

Also $B^{*}$ is the optimal parameter vector as: 


$$
B^{*} \stackrel{\Delta}{=} \arg \min _{B}\left\{\left|B^{T} W-u^{*}\right|\right\}
$$

\subsection{Adaptive fuzzy sliding mode control}

In practice, the optimal parameter vector $B^{*}$ such as the uncertainty bound $\Psi$, may be unknown. If $\hat{u}_{f u z}(s, \hat{B})$ be a fuzzy system for the approximation of $u^{*}$, as:

$$
\hat{u}_{f u z}(s, \hat{B})=\hat{B}^{T} W
$$

where $\hat{B}$ is the approximated value of $B^{*}$, then the control law for the AFSMC system, becomes:

$$
u=\hat{u}_{f u z}(s, \hat{B})+u_{r b}(s)
$$

where the fuzzy controller $\hat{u}_{f u z}$ is designed for approximating the ideal controller $u^{*}$, and the robust controller $u_{r b}$ is designed for compensating the difference between the ideal controller and the fuzzy controller.

By defining the approximation errors as:

$$
\begin{gathered}
\tilde{u}_{f u z}=u^{*}-\hat{u}_{f u z} \\
\tilde{B}=B^{*}-\hat{B} \\
\tilde{\Psi}(t)=\Psi-\hat{\Psi}(t)
\end{gathered}
$$

and using (9) and (12), gives:

$$
\tilde{u}_{f u z}=\tilde{B}^{T} W+\psi
$$

If the AFSMC system is designed according to (13) and the parameter vector of the fuzzy system is adaptively tuned according to :

$$
\dot{\hat{B}}=-\dot{\tilde{B}}=\alpha_{1} s(t) W
$$

and the robust controller is designed using:

$$
u_{r b}=\hat{\Psi} \operatorname{sgn}[s(t)] \operatorname{sgn}(g)
$$

and the bound estimation algorithm given by:

$$
\dot{\hat{\Psi}}=-\dot{\tilde{\Psi}}=\alpha_{2}|s(t)| \operatorname{sgn}(g)
$$

where $\alpha_{1}$ and $\alpha_{2}$ are the learning rates chosen by the user, then the stability of the AFSMC system will be guaranteed $[8,9]$. The block diagram of the AFSMC algorithm has been shown in Fig. 1. 


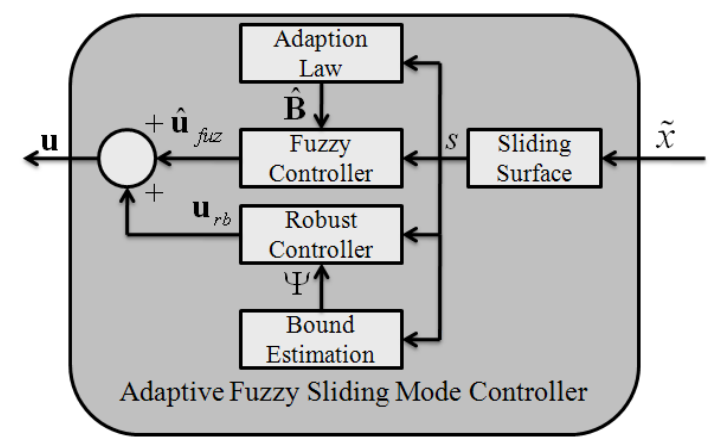

Fig. 1: Block diagram of the AFSMC algorithm.

\section{System modeling}

The performance of the AFSMC algorithm under semi-active approaches of AFSMC/Clipped is demonstrated by numerical simulation. A three-story building model which is equipped with one MR damper in the first floor is considered as shown in Fig. 2. The MR damper is rigidly placed between the ground and the first floor of the building. The building dynamic equations of motion can be written as

$$
M \ddot{\mathbf{x}}+C \dot{\mathbf{x}}+K \mathbf{x}=\Gamma \mathbf{f}-M \Lambda \ddot{x}_{g}
$$

where, $\mathbf{x}=\left[x_{1}, x_{2}, x_{3}\right]^{T}, \dot{\mathbf{x}}=\left[\dot{x}_{1}, \dot{x}_{2}, \dot{x}_{3}\right]^{T}$ and $\ddot{\mathbf{x}}=\left[\ddot{x}_{1}, \ddot{x}_{2}, \ddot{x}_{3}\right]^{T}$ are the vectors of displacement, velocity and acceleration of the building floors relative to the ground respectively and $\mathbf{f}=\left[f_{M R}, 0,0\right]^{T}$ is the vector of the control force. Moreover $\Gamma$ is the vector of control force position, $\Lambda$ is the vector of perturbation signals, $M$ is the mass matrix, $C$ is the damping matrix and $K$ is the stiffness matrix, which have been given as:

$$
\Gamma=\left[\begin{array}{c}
-1 \\
0 \\
0
\end{array}\right] \Lambda=\left[\begin{array}{l}
1 \\
1 \\
1
\end{array}\right] M=\left[\begin{array}{ccc}
98.3 & 0 & 0 \\
0 & 98.3 & 0 \\
0 & 0 & 98.3
\end{array}\right] K g \quad C=\left[\begin{array}{ccc}
175 & -50 & 0 \\
-50 & 100 & -50 \\
0 & -50 & 50
\end{array}\right] \frac{N . s e c}{m} \quad K=10^{5}\left[\begin{array}{ccc}
12 & -6.84 & 0 \\
-6.84 & 13.7 & -6.84 \\
0 & -6.84 & 6.84
\end{array}\right] \frac{N}{m}
$$

In this study, a phenomenological model for the SD-1000 MR damper of the LORD company is used; for which the parameters are given by Spencer et al. [10]. The structure model is subjected to the North-South (NS) component of the recorded ground acceleration for the 1940 EL CENTRO earthquake. It should be mentioned that for the simulation purpose, the earthquake is reproduced at five times the actual recorded rate. 


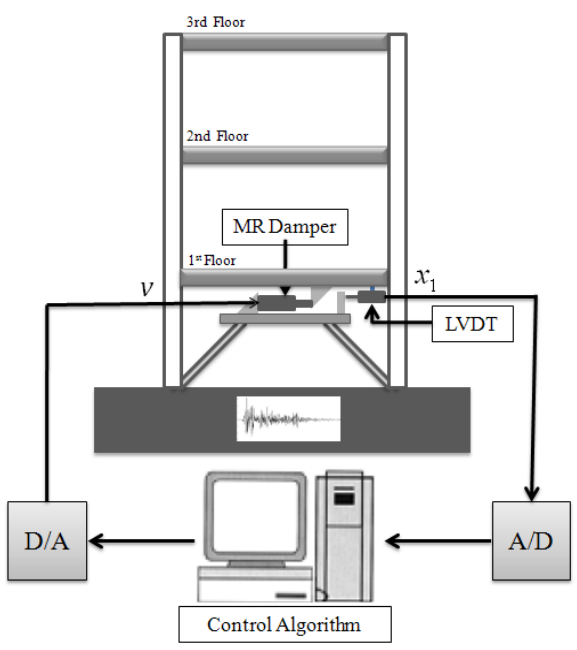

Fig. 2: Schematic diagram of a three-story building equipped with a MR damper.

The AFSMC controller determines the needed control force, and an internal force-following loop approximately generates the required interaction force by intermittent activation of the semi-active dampers (Clipped algorithm). Now, the equations of motion (21) for matching with systemError! Reference source not found. are rewritten as:

$$
x_{1}^{(2)}=f(\mathbf{x})+g(\mathbf{x}) u
$$

where, $\mathrm{x}$ is the state vector of the system, $u$ is the force calculated by the controller, i.e. $u=f_{A F S M C}$ and $x_{1}$ is the displacement of the first floor relative to the ground. The objective is to control the displacement and acceleration of the building floors. For this purpose, the sliding surface is defined as:

$$
s=\dot{\tilde{x}}_{1}+\lambda \tilde{x}_{1}
$$

with $\lambda=3$. The input membership functions for $s$ are chosen as illustrated in Fig. 3. The initial output membership functions are arbitrarily chosen as $\hat{B}=[-8,0,8]$, and the initial value of uncertainty bound is selected as $\Psi=0.1$. The learning rates are adjusted to $\alpha_{1}=512$ and $\alpha_{2}=2315$. Following the simulation, the final output membership functions are obtained as $\hat{B}=[-217,0,190]$ and the final value of uncertainty bound is $\Psi=2750$. After the computing of control force, the best candidate for conversion is the Clipped algorithm developed by Dyke et al. [1] as:

$$
v=V_{\max } H\left\{\left(f_{A F S M C}-f_{M R}\right) f_{M R}\right\}
$$

where, $v$ is the applied voltage level, $V_{\max }$ is the maximum voltage level, $H(\cdot)$ is the heaviside step function, and $f_{M R}$ is the output force generated by the MR damper. The block diagram of the semi-active AFSMC/Clipped system is shown in Fig. 4. 


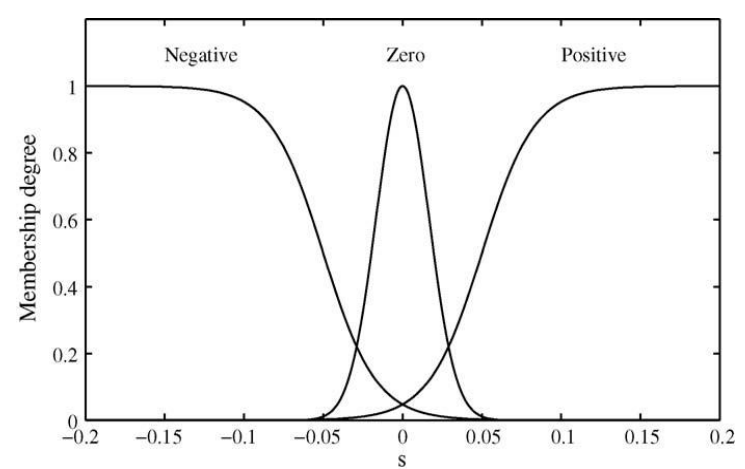

Fig. 3: Input membership functions of s for AFSMC systems.

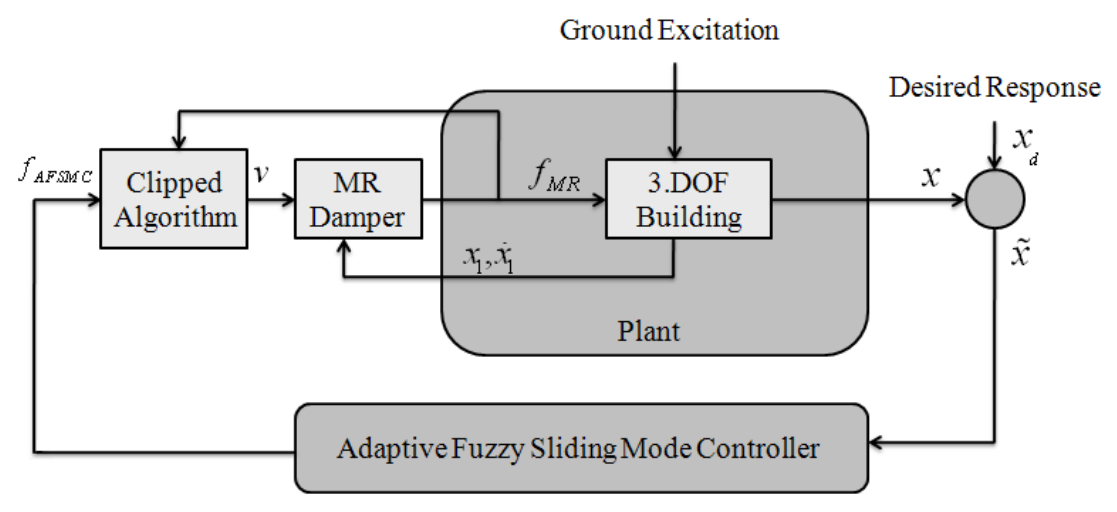

Fig. 4: Block diagram of the semi-active AFSMC/Clipped system.

\section{Results}

The maximum building responses to the EL-Centro earthquake for the uncontrolled case and AFSMC/Clipped system are shown in table 1 . Here, $X_{i}$ is the maximum displacement of the $i$ th floor relative to the ground, $D_{i}$ is the maximum inter-story drift (i.e. $X_{i}-X_{i-1}$ ), $\ddot{X}_{a i}$ is the maximum absolute acceleration of the $i$ th floor, and $f$ is the maximum applied control force. Moreover, for the sake of comparison, the results given by H2-LQG/Clipped control strategy [1] have been listed in Table 1. It should be noted that, the H2-LQG/clipped control strategy is the most referred semi-active algorithms applied on three-story building and any comparison with this method determines the excellence of applied approaches.

Table 1: Maximum building responses due to the 1940 El-Centro earthquake.

\begin{tabular}{|c|c|c|c|}
\hline 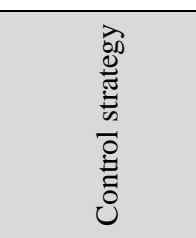 & 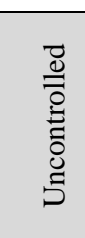 & 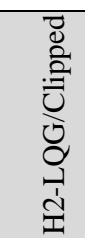 & 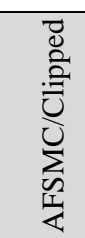 \\
\hline$X_{i}(\mathrm{~cm})$ & $\begin{array}{l}0.538 \\
0.820 \\
0.962\end{array}$ & $\begin{array}{l}0.114 \\
0.185 \\
0.212\end{array}$ & $\begin{array}{l}0.073 \\
0.182 \\
0.266\end{array}$ \\
\hline$D_{i}(\mathrm{~cm})$ & $\begin{array}{l}0.538 \\
0.319 \\
0.201\end{array}$ & $\begin{array}{c}0.114 \\
0.09 \\
0.101\end{array}$ & $\begin{array}{l}0.076 \\
0.140 \\
0.090\end{array}$ \\
\hline$\ddot{X}_{a i}\left(\mathrm{~cm} / \mathrm{sec}^{2}\right)$ & $\begin{array}{c}856 \\
1030 \\
1400\end{array}$ & $\begin{array}{l}696 \\
739 \\
703\end{array}$ & $\begin{array}{l}288 \\
473 \\
673\end{array}$ \\
\hline$f(N)$ & - & 941 & 863 \\
\hline
\end{tabular}


Fig. 5 shows the time responses of the third floor of the building for the AFSMC/Clipped approach due to the 1940 EL-Centro earthquake. As Table 1 and Fig. 5 indicate, the AFSMC system have been able to reduce the displacement and absolute acceleration responses of the entire building floors quite well compared to the uncontrolled case.

Fig. 6 compares the maximum building responses for the uncontrolled case, the AFSMC/Clipped systems and the H2LQG/Clipped control strategy. Diagram (a) compares the maximum relative displacement, (b) compares the maximum inter-story drift and (c) compares the maximum absolute acceleration of the floors. As illustrated, for the case of maximum displacements, the AFSMC/Clipped demonstrates a better performance at the first and second floors, while, the H2LQG/Clipped method surpasses the AFSMC/Clipped method at the third floor. As shown, AFSMC/Clipped and the H2LQG/Clipped controllers have been able to reduce the maximum displacements by average values of $79 \%$ and $78 \%$, respectively.
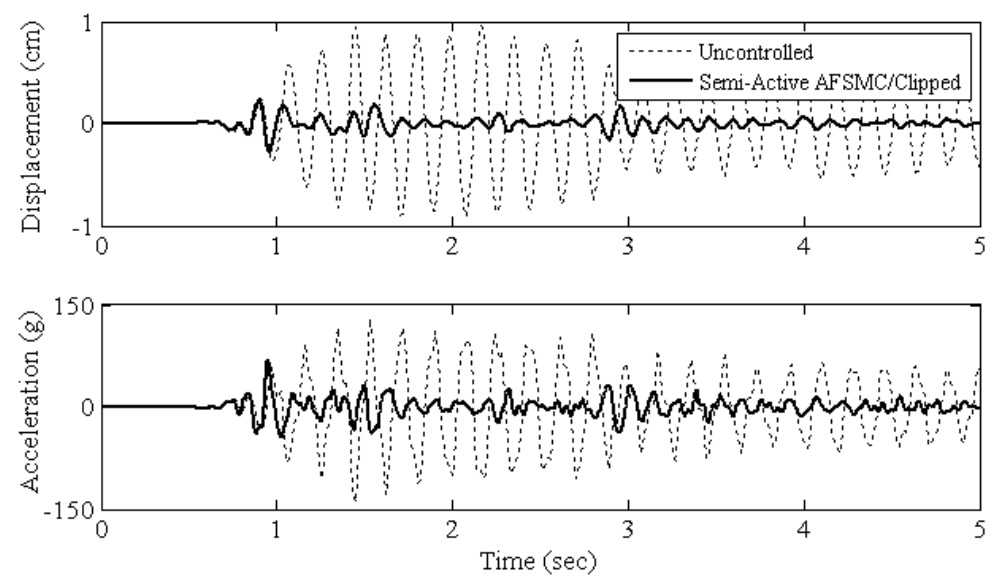

Fig. 5: Time responses of the third floor of the building for the semi-active AFSMC/Clipped approach.

There is a close competition between the controllers for the maximum inter-story drift, and both could be considered as equivalent performers. For the case of maximum acceleration of floors, although both controllers depict similar results at the third floor, the AFSMC/Clipped shows its superiority at the first and second floors. In particular, the AFSMC/Clipped and the H2-LQG/Clipped controllers have been able to reduce the maximum absolute acceleration by an average of $57 \%$ and $32 \%$, respectively. Therefore, in the competition between AFSMC/Clipped and H2-LQG/Clipped methods, the proposed AFSMC/Clipped wins, for the former reduces the acceleration considerably more than the latter while the expenditure of control energy through the MR damper is also kept at a lower value. Fig. 7 shows the MR Damper output force for the AFSMC/Clipped approach.
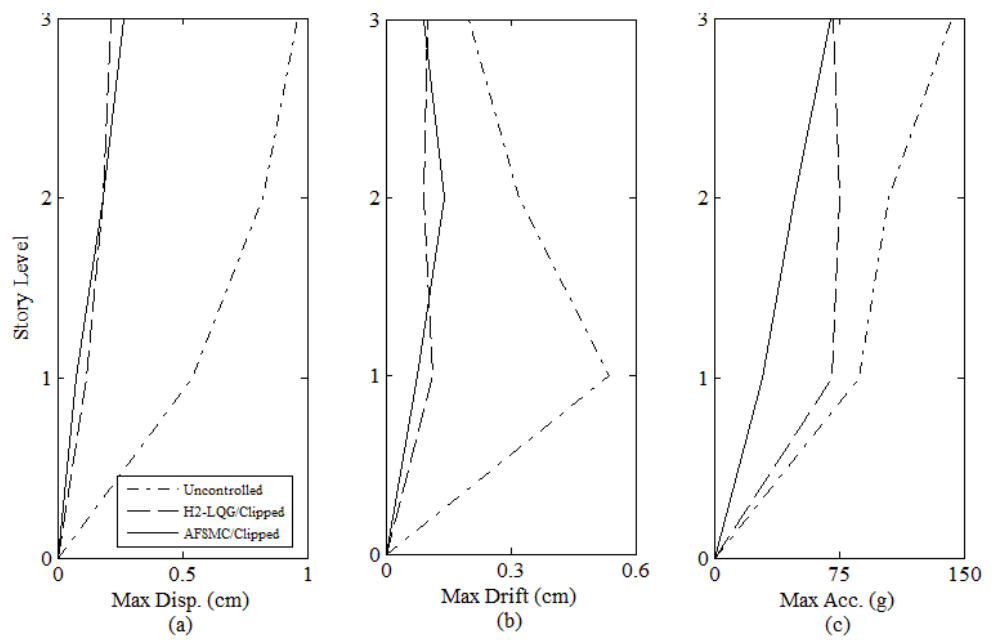

Fig. 6: Comparison of maximum building responses in the uncontrolled case, AFSMC/Clipped and H2-LQG/Clipped systems. 


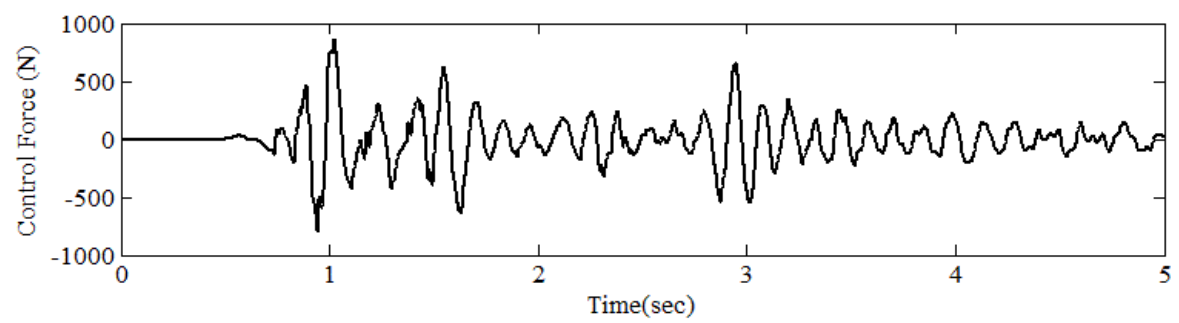

Fig. 7: MR damper output force for AFSMC/Clipped approach.

\section{Conclusion}

In this paper, a new adaptive fuzzy sliding mode control algorithm was employed for semi-active control of earthquake-induced excitations. By applying the AFSMC controller on a three-story benchmark building, it was revealed that by using the AFSMC algorithm instead of the H2-LQG strategy and then by converting the force signal into the MR damper input voltage, the induced vibrations can be reduced considerably, while the control effort is also reduced. The importance of this result is even more highlighted, considering the fact that proposed control method does not use any of the model parameters for the calculation of the required control force. The AFSMC/Clipped strategy have been able to reduce the displacement and absolute acceleration responses of the entire building floors quite well compared to the uncontrolled case. Also, for the case of maximum displacements, the AFSMC/Clipped demonstrates a better performance at the first and second floors, while, the H2-LQG/Clipped method exceeds the AFSMC/Clipped method at the third floor. Furthermore, there is a close competition between the controllers for the maximum inter-story drift, and both could be considered as equivalent performers. For the case of maximum acceleration of floors, although both controllers depict similar results at the third floor, the AFSMC/Clipped shows its superiority at the first and second floors.

\section{Nomenclature}

$\tilde{b}^{r} \quad$ Fuzzy singleton for the output of the $r$ th rule

$d \quad$ Lumped uncertainty

$f \quad$ Maximum applied control force

f Vector of the control force

$f_{\text {AFSMC }}$ Active AFSMC force

$f_{M R} \quad$ MR damper output force

$n_{r} \quad$ Fuzzy rules number

$s \quad$ Sliding surface

$u \quad$ Control input

$u^{*} \quad$ Ideal controller

$u_{e q} \quad$ Equivalent Sliding mode controller

$u_{f u z} \quad$ Fuzzy controller

$\hat{u}_{f u z} \quad$ Approximation of fuzzy controller

$\tilde{u}_{f u z} \quad$ Approximation error of fuzzy controller

$u_{f u z}^{*} \quad$ Ideal fuzzy controller

$u_{r b} \quad$ Robust controller

$v \quad$ MR damper input voltage

$w^{n_{r}} \quad$ Firing strength of the $r$ th rule

$\mathbf{X}$
Vectors of displacement relative to the ground

$\begin{array}{ll}\dot{\mathbf{x}} & \text { Vectors of velocity relative to the ground } \\ \ddot{\mathbf{x}} & \text { Vectors of acceleration relative to the ground } \\ \tilde{x} & \text { Tracking error } \\ x_{d} & \text { Desired trajectory of system states } \\ \tilde{A}^{r} & \text { Fuzzy set of the } r \text { th rule } \\ B & \text { Fuzzy parameter vector } \\ \hat{B} & \text { Approximation of parameter vector } \\ \tilde{B} & \text { Approximation error of parameter vector } \\ B^{*} & \text { Fuzzy optimal parameter vector } \\ C & \text { Damping matrix } \\ D_{i} & \text { Maximum inter-story drift of the } i \text { th floor } \\ K & \text { Stiffness matrix } \\ M & \text { Mass matrix } \\ V_{i} & \text { Lyapunov function } \\ V_{\text {max }} & \text { MR damper maximum voltage } \\ W & \text { Vector of rules weight } \\ X_{1} & \text { Maximum displacement of the } i \text { th floor } \\ & \text { Maximum absolute acceleration of the } i \text { th floor } \\ & \end{array}$




$\begin{array}{llll}\alpha_{2} & \text { Second learning rate of AFSMC } & \Lambda & \text { Vector of perturbation signals } \\ \Gamma & \text { Vector of control force position } & \psi & \text { Approximation error } \\ \delta & \text { Uncertainty bound } & \Psi & \text { Approximation error bound } \\ \lambda & \text { User defined constant } & & \end{array}$

\section{References}

[1] S. J. Dyke, B. F. Spencer, M. K. Sain, J. D. Carlson, "Modeling and control of magnetorheological dampers for seismic response reduction," Smart Mater. Struct., vol. 5, no. 5, pp. 565-575, 1996.

[2] N. Aguirre, F. Ikhouane, J. Rodellar, "Proportional-plus-integral semiactive control using magnetorheological dampers," J. Sound Vib., vol. 330, no. 10, pp. 2185-2200, 2011.

[3] T. E. Alqado, G. Nikolakopoulos, "Posicast control of structures using MR dampers," Struct. Control. Health Monit., 2016.

[4] M. Askari, A. H. D. Markazi, "A new evolving compact optimised Takagi-Sugeno fuzzy model and its application to nonlinear system identification," Int. J. Syst. Sci., vol. 43, no. 4, pp. 776-785, 2012.

[5] H. S. Kim, "Seismic response control of adjacent buildings coupled by semi-active shared TMD," Int. J. Steel Struct., vol. 16, no. 2, pp. 647-656, 2016.

[6] L. X. Wang, Adaptive fuzzy systems and control: design and stability analysis. New Jersey: Prentice-Hall, 1994.

[7] Y. C. Fan, C. H. Loh, "Experimental performance evaluation of an equipment isolation using MR dampers," Earthq. Eng. Struct. D., vol. 38, no. 3, pp. 285-305, 2009.

[8] A. Poursamad, A. H. D. Markazi, "Rubust adaptive fuzzy control of unknown chaotic systems," Appl. Soft Comput., vol. 9, no. 3, pp. 970-976, 2009.

[9] A. Poursamad, A. H. D. Markazi, "Adaptive fuzzy sliding-mode control for multi-input multi-output chaotic systems," Chaos Solitons Fractals., vol. 42, no. 5, pp. 3100-3109, 2009.

[10] B. F. Spencer, J. Dyke, M. K. Sain, J. D. Carlson, "Phenomenological Model of a Magnetorheological Damper," J. Eng. Mech., vol. 123, pp. 230-238, 1997. 